\title{
scikit-posthocs: Pairwise multiple comparison tests in Python
}

\author{
Maksim A. Terpilowski ${ }^{1}$ \\ 1 Institute of Evolutionary Physiology and Biochemistry, Saint Petersburg, Russia
}

DOI: $10.21105 /$ joss. 01169

\section{Software}

- Review ct

- Repository ๘

- Archive ${ }^{\top}$

Submitted: 06 December 2018

Published: 14 April 2019

\section{License}

Authors of papers retain copyright and release the work under a Creative Commons Attribution 4.0 International License (CC-BY).

\section{Summary}

scikit-posthocs is a Python package providing multiple pairwise comparison tests (post hocs). Statisticians, data scientists, and researchers will find it useful in a statistical analysis routine to assess the differences between group levels if a statistically significant result of a parametric or nonparametric analysis of variance (ANOVA) test has been obtained.

Most statistical software packages (such as SPSS and Stata) provide a number of post hoc tests. However, Python ecosystem still lacks implementation of these tests for factorial and block design data. Currently, Student, Wilcoxon, Mann-Whitney tests which are not adapted to multiple pairwise comparisons are offered by SciPy. Statsmodels package includes TukeyHSD test which needs extra actions to be fluently integrated into a data analysis pipeline.

scikit-posthocs package is aimed at filling this gap by providing a number of nonparametric and parametric pairwise comparisons tests as well as outliers detection algorithms implemented in Python.

Currently, the following tests are implemented in this package:

1. Parametric pairwise multiple comparisons tests: Scheffe, Student T, Tamhane T2, and TukeyHSD test.

2. Non-parametric tests for factorial design: Conover, Dunn, Dwass-Steel-CritchlowFligner, Mann-Whitney, Nashimoto-Wright (NPM), Nemenyi, van Waerden, and Wilcoxon test.

3. Non-parametric tests for block design: Conover, Durbin and Conover, Miller, Nemenyi, Quade, Siegel test.

4. Additional tests: Anderson-Darling, Mack-Wolfe, Hayter (OSRT) test.

scikit-posthocs provides tests for outliers detection: interquartile range (IQR) test, Grubbs test, Tietjen-Moore test, and generalized (extreme Studentized deviate) ESD test. It also has plotting functionality to present the results of pairwise comparisons as a heatmap (significance plot, see figure).

scikit-posthocs is compatible with Python 2 and 3 versions, relies heavily and extends the functionality of statsmodels, SciPy and PMCMRplus packages (Seabold \& Perktold, 2010), (Jones, Oliphant, \& Peterson, 2001), (Pohlert, 2018). It is also integrated with Pandas (McKinney, 2010) and Numpy (T. E. Oliphant, 2006) for efficient computations and data analysis. The package is fully documented and comes with a Jupyter notebook example. 


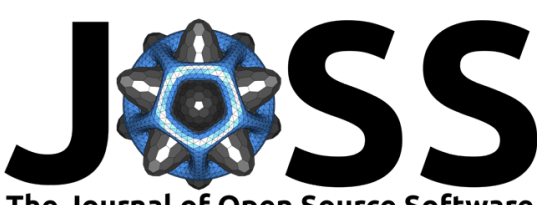

The Journal of Open Source Software

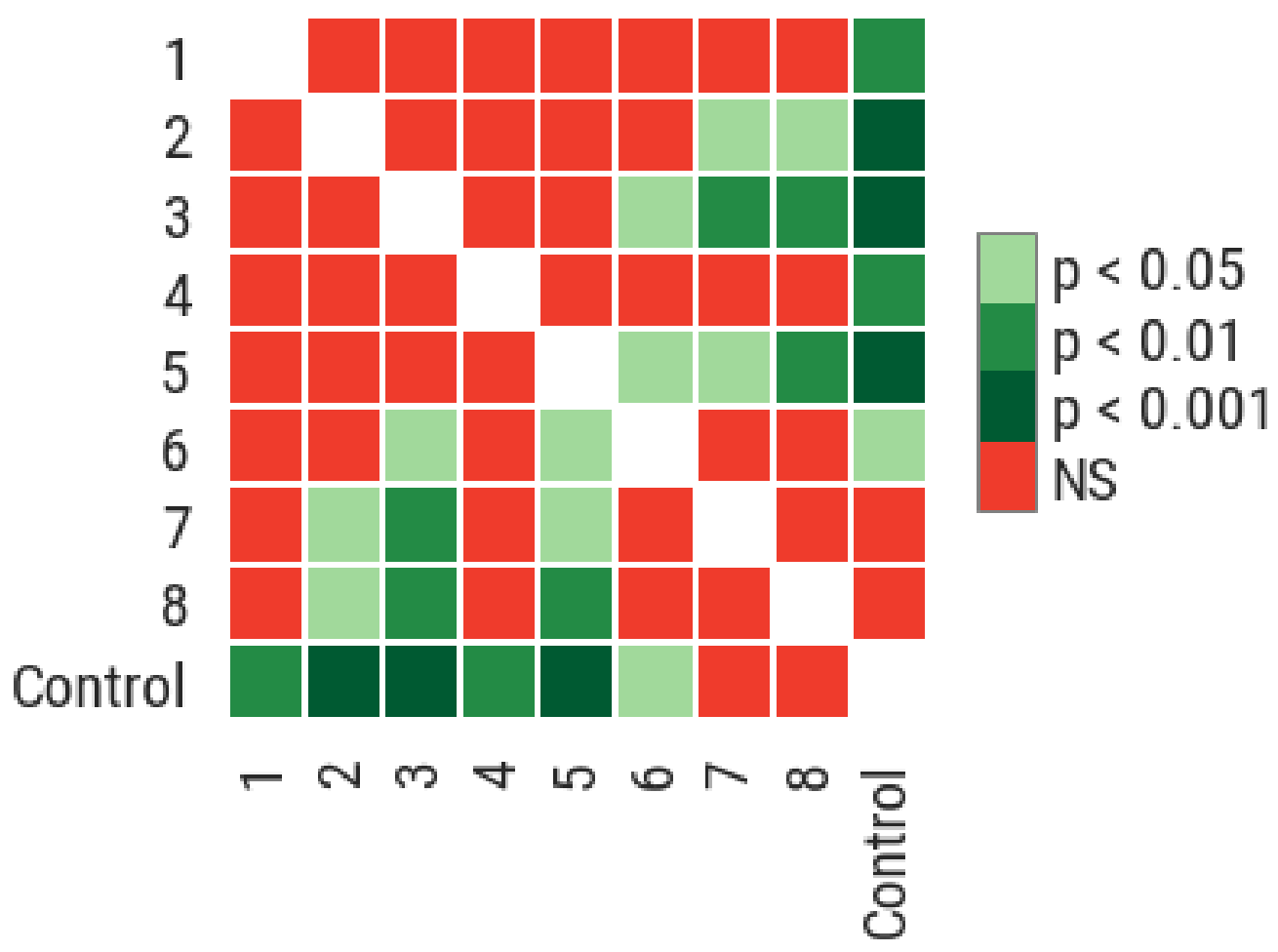

Figure 1: Significance plot

\section{References}

Jones, E., Oliphant, T., \& Peterson, P. (2001). SciPy: Open source scientific tools for python. Retrieved from http://www.scipy.org/

McKinney, W. (2010). Data structures for statistical computing in python. In Proceedings of the 9th python in science conference (Vol. 445, pp. 51-56). Austin, TX.

Oliphant, T. E. (2006). A guide to numpy (Vol. 1). Trelgol Publishing USA.

Pohlert, T. (2018). PMCMRplus: Calculate pairwise multiple comparisons of mean rank sums extended. Retrieved from https://CRAN.R-project.org/package=PMCMRplus

Seabold, S., \& Perktold, J. (2010). Statsmodels: Econometric and statistical modeling with python. In Proceedings of the 9th python in science conference (Vol. 57, p. 61). SciPy society Austin. 